\title{
Ultrasound ablation enhances drug accumulation and survival in mammary carcinoma models
}

\author{
Andrew W. Wong, ${ }^{1}$ Brett Z. Fite, ${ }^{1}$ Yu Liu, ${ }^{1}$ Azadeh Kheirolomoom, ${ }^{1}$ Jai W. Seo, ${ }^{1}$ Katherine D. Watson, ${ }^{1}$ Lisa M. Mahakian, ${ }^{1}$ \\ Sarah M. Tam, ${ }^{1}$ Hua Zhang, ${ }^{1}$ Josquin Foiret, ${ }^{1}$ Alexander D. Borowsky, ${ }^{2}$ and Katherine W. Ferrara' \\ 'Department of Biomedical Engineering and ²Center for Comparative Medicine, University of California, Davis, California, USA.
}

\begin{abstract}
Magnetic resonance-guided focused ultrasound (MRgFUS) facilitates noninvasive image-guided conformal thermal therapy of cancer. Yet in many scenarios, the sensitive tissues surrounding the tumor constrain the margins of ablation; therefore, augmentation of MRgFUS with chemotherapy may be required to destroy remaining tumor. Here, we used ${ }^{64} \mathrm{Cu}-\mathrm{PET}-\mathrm{CT}$, MRI, autoradiography, and fluorescence imaging to track the kinetics of long-circulating liposomes in immunocompetent mammary carcinoma-bearing FVB/n and BALB/c mice. We observed a 5-fold and 50-fold enhancement of liposome and drug concentration, respectively, within MRgFUS thermal ablation-treated tumors along with dense accumulation within the surrounding tissue rim. Ultrasound-enhanced drug accumulation was rapid and durable and greatly increased total tumor drug exposure over time. In addition, we found that the small molecule gadoteridol accumulates around and within ablated tissue. We further demonstrated that dilated vasculature, loss of vascular integrity resulting in extravasation of blood cells, stromal inflammation, and loss of cell-cell adhesion and tissue architecture all contribute to the enhanced accumulation of the liposomes and small molecule probe. The locally enhanced liposome accumulation was preserved even after a multiweek protocol of doxorubicin-loaded liposomes and partial ablation. Finally, by supplementing ablation with concurrent liposomal drug therapy, a complete and durable response was obtained using protocols for which a sub-mm rim of tumor remained after ablation.
\end{abstract}

\section{Introduction}

Magnetic resonance-guided focused ultrasound (MRgFUS) provides completely noninvasive thermal therapy for cancer. To address systemic cancers, such local therapies are augmented with chemotherapy or immunotherapy. In addition, at tumor margins and near blood vessels or vulnerable structures, practical ablative protocols may not be fully effective. Thus, there are multiple motivations and precedents for combining systemic and local therapies; for example, radiation therapy combined with neoadjuvant chemotherapy reduces risk of local recurrence in breast-conserving surgery $(1,2)$. Furthermore, the efficacy of radiofrequency (RF) ablation is improved by preablation thermosensitization (3) and coadministration of ablation and chemotherapy (4-6), suggesting that combining ablation with drug therapy may also increase coagulation diameter and reduce recurrence using MRgFUS. The purpose of this study is to determine whether a practical ablation strategy in which the tumor margin is incompletely treated to preserve sensitive surrounding tissue can be locally curative in mammary carcinoma.

MRgFUS ablation involves the application of a pattern of ablation foci across the desired volume of interest. Clinical strategies for ablation of large regions are realized by scanning the ultrasound focus in a circular pattern or creating a grid pattern of overlapping ablation points $(7,8)$. Treatment is monitored by magnetic resonance thermometry, which typically makes use of the temperature dependence of the proton resonance frequency $(9,10)$. This can be

Conflict of interest: The authors have declared that no conflict of interest exists Submitted: June 18, 2015; Accepted: October 9, 2015.

Reference information: / Clin Invest. 2016;126(1):99-111. doi:10.1172/JCI83312. used to generate maps of thermal dose with the thermal isoeffect measure cumulative equivalent minutes at $43^{\circ} \mathrm{C}$ (CEM43) (11). As CEM43 increases rapidly with temperature, high temporal resolution is particularly important for ablative strategies that utilize high temperature and short heating time, but minimize toxicity to surrounding tissue (12). By modeling the thermal dose and comparing this to the experimental results, the effect of ablation on delivery can be assessed at microscopic and macroscopic scales both within and surrounding the ablated region. The impact of such scanning protocols on coadministered chemotherapeutics has not been evaluated in previous studies. Single-point and grid insonation patterns have been proposed as protocols for enhanced drug delivery or immunotherapy, where treatment can be repeated and potentially combined with adjuvants $(13,14)$. Complete ablation can also be achieved with circular insonation or tightly spaced grid protocols.

We used small animal MRI and nuclear imaging to track the biodistribution and pharmacokinetics of small molecules and nanoparticle therapy, respectively, in conjunction with MRgFUS. Integration of ${ }^{64} \mathrm{Cu}$ PET labels within liposomes was achieved by the use of 6 -[ $p$-(bromoacetamido)benzyl]-1,4,8,11tetraazacyclotetradecane- $N, N^{\prime}, N^{\prime \prime}, N^{\prime \prime \prime}$-tetraacetic acid (BAT), which demonstrates excellent stability in vitro and in vivo (15). The ${ }^{64} \mathrm{Cu}$ radiotracer is particularly appropriate for labeling nanoparticles, as its half-life of 12.7 hours is comparable to the circulating half-life of long-circulating liposomes (LCL).

A mechanistic understanding of the interaction between ablation and chemotherapeutic delivery is key to improving therapy. Combining ablation with chemotherapy has been hypothesized to improve the therapeutic outcome by enhancing delivery and 
lethality in regions of sublethal hyperthermia (11). Hyperthermia enhances local accumulation of nanoparticles due to enhanced blood flow, changes in the effective pore size of vasculature, and release of inflammatory and vasodilatory substances from ablated mast cells or endothelial cells $(4-6,16-20)$. While ultrasoundinduced mild hyperthermia alone can enhance the accumulation of nanotherapeutics, the accumulation has been shown to be dependent on the tumor phenotype (21). Moreover, the use of an ablation protocol is attractive, as it provides the opportunity to debulk large tumors while enhancing accumulation. Here, we test whether such a strategy enhances accumulation in multiple tumor cell phenotypes. Further, burns are known to induce the release of factors such as histamine and VEGF, which can induce local edema and enhance accumulation of nanoparticles (17-20, 22). Thermal injury can also reduce interstitial fluid pressure (23), which has been shown to enhance accumulation of nanoparticles in cancer (21), and modulate immune cell migration and oxygen tension (24-26). Unlike other modes of thermal therapy, ultrasound also induces mechanical tissue effects (27), which enhance local accumulation of drugs, antibodies, and nanoparticles (28-30). Mechanisms for these changes include radiation force, microstreaming, and oscillating gas bubbles within tissue and vasculature (31).

Nanoparticle formulation improves efficacy of systemic chemotherapy. Encapsulation of chemotherapeutics within liposomes enhances solubility, reduces systemic toxicity, prevents degradation, and changes drug pharmacokinetics (32). Most longcirculating nanoparticle formulations (including LCL) accumulate within tumors due to the enhanced permeability and retention (EPR) effect (33); however, EPR-mediated accumulation varies with tumor site and is often heterogeneous. Therefore, enhancing this effect is desirable. Alternatively, activatable nanotherapeutics release their cargo in response to changes in $\mathrm{pH}$, temperature, or other stimuli (12). Temperature-sensitive liposomes (TSL) have been paired with ultrasound hyperthermia $(34,35)$ and shown to increase drug accumulation in the insonified tumor. Temperaturesensitive formulations were less effective than long-circulating drug carriers when combined with RF ablation (36); however, few data are available on the combination of TSL and ultrasoundmediated ablation.

This study makes use of 2 syngeneic, orthotopic breast cancer models: the neu exon deletion line (NDL) model of epithelial, focal, mammary adenocarcinoma and the $4 \mathrm{~T} 1$ model of invasive and metastatic mammary adenocarcinoma. NDL tumors were generated in $\mathrm{FVB} / \mathrm{n}$ mice expressing an activated form of ERBB2/ neu with the mouse mammary tumor virus long-terminal repeat promoter, a system modeling human HER2-amplified breast cancer $(37,38)$. These tumors were then transplanted into the mammary fat pads of wild-type (WT) FVB mice. 4T1 cells were purchased from ATCC and injected into the mammary fat pads of WT $\mathrm{BALB} / \mathrm{c}$ mice. Use of this cell line in an orthotopic model provides a highly proliferative model of breast cancer with an increase in the EPR effect relative to the NDL model.

Our ultimate goal is to use MRgFUS within curative protocols. Curing mice with ultrasound ablation is challenging, as the skin is highly absorptive and is near the tumor; also, critical organs are nearby. Thus, the mouse is a useful model for evaluating strategies to augment ablation with drug delivery near sensitive structures and to determine where delivery is enhanced. We first applied ultrasound to a single point at the center of the tumor to assess the surrounding nanoparticle accumulation, with temperatures greater than $65^{\circ} \mathrm{C}$. This protocol recapitulates direct thermal toxicity at the tumor center, surrounded by a shell of sublethal hyperthermia, where we expected enhanced nanoparticle accumulation. We also applied a scanned circular pattern and a grid protocol of ultrasound exposure in order to quantify nanoparticle accumulation within these ablated regions and the surrounding rim. We assessed the sequestration of nanoparticles on a macroand microscopic basis and used contrast-enhanced T1-weighted MRI (CET1wMRI) to compare this with the accumulation of the small molecule gadoteridol following MRgFUS in a mouse model of mammary carcinoma. We further explored whether ultrasound-mediated enhancement of accumulation persisted through multiple applications of ablation and chemotherapy. Finally, the combination of ablation and chemotherapy delivery was shown to enhance survival in this model system.

\section{Results}

MRgFUS accurately controlled the desired temperature increase within each protocol. We first set out to apply PET-CT to assess drug accumulation over 48 hours after ablation and LCL injection in the NDL tumor model (Figure 1A). The spatial distribution of heat was mapped and controlled by MRgFUS (Figure 1, B-F), and the instantaneous verbal map was used to visualize the region treated in the single-point (Figure 1, B and E), grid (Figure 1, D and E), or circular protocol (Figure 1, C and F). For the grid protocol, individual treatment points were sequentially directed to the tumor and the region was allowed to cool between insonations (Figure 1D); therefore, the instantaneous thermal map was identical to that for the single-point insonation (Figure 1E). We also simulated the temperature increase for our parameters in order to ensure that values obtained by MR thermometry matched the conditions (Figure 1, G-J). In each study, thermometry validated a peak temperature greater than $65^{\circ} \mathrm{C}$ and a CEM 43 exceeding 5,000 .

With the application of a single ablation point, the spatial extent of an instantaneous temperature above $55^{\circ} \mathrm{C}$ was predicted and measured to be 1 to $2 \mathrm{~mm}$ in radius (Figure $1 \mathrm{G}$ and Supplemental Figure 1, A and B; supplemental material available online with this article; doi:10.1172/JCI83312DS1) and approximately 3 $\mathrm{mm}$ in depth. For the scanned (circle) ablation, the extent of the $55^{\circ} \mathrm{C}$ temperature was approximately $5 \mathrm{~mm}$ in radius and depth, resulting in a greater deposition of heat (Figure $1 \mathrm{H}$ ) and expanded region of cell death (Supplemental Figure 1C). The experimentally observed distribution corresponded well with simulations of the ultrasound beam and heat deposition, with small differences noted in the spatial extent and magnitude of heating (Figure 1, E-J). Therefore, we find that MRgFUS can reproducibly create the desired local thermal dose.

PET-CT confirmed the enhanced accumulation within the ablated NDL tumors. The effect of MRgFUS on ${ }^{64} \mathrm{Cu}-\mathrm{LCL}$ distribution was confined to the region of insonation and immediately surrounding tissue. Without MRgFUS ablation, PET-CT imaging of mice with bilateral NDL tumors at 3 hours after injection with ${ }^{64} \mathrm{Cu}-\mathrm{LCL}$ revealed a vascular distribution of liposomes, with greater activity in the heart, jugular veins, and liver (Figure 1K). At 20 and 48 
A

\begin{tabular}{|c|c|}
\hline $0 \mathrm{wk}$ & Implant \\
\hline $3 \mathrm{wk}$ & $\begin{array}{l}\text { MRI (pre \& post ablati } \\
\text { Ablation or control (no } \\
\text { Inject }{ }^{64} \mathrm{Cu} \text {-LCL IV }\end{array}$ \\
\hline $\begin{array}{r}3 \mathrm{wk}+ \\
1.5 \text { to } 48 \mathrm{~h}\end{array}$ & $\begin{array}{l}\text { MRI }(1.5 \text { to } 6 h) \\
\text { PET/CT ( } 3 \text { to } 48 \text { h) }\end{array}$ \\
\hline $\begin{array}{r}3 w k+ \\
48 \mathrm{~h}\end{array}$ & $\begin{array}{l}\text { Histology \& } \\
\text { autoradiography }\end{array}$ \\
\hline
\end{tabular}

$\mathbf{K}$

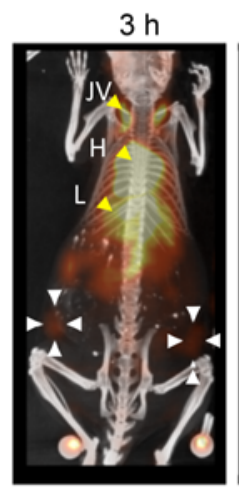

No US

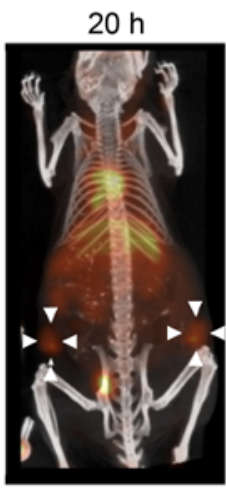

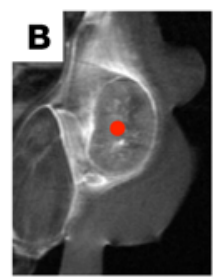

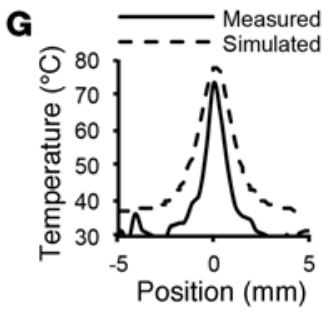

$\mathbf{L}$

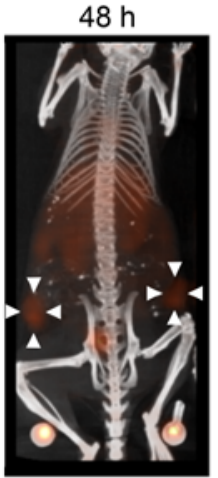

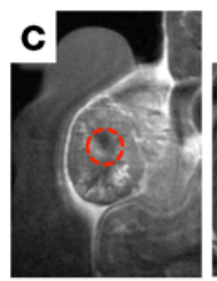
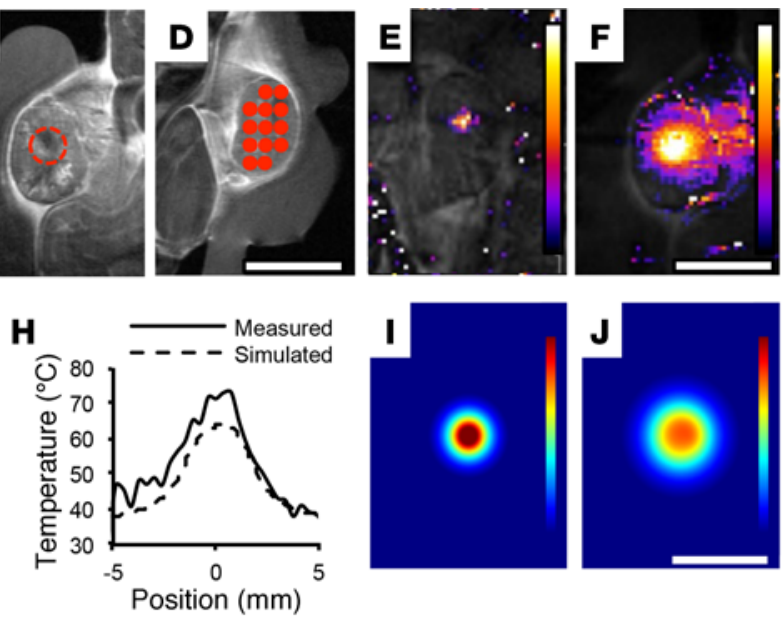
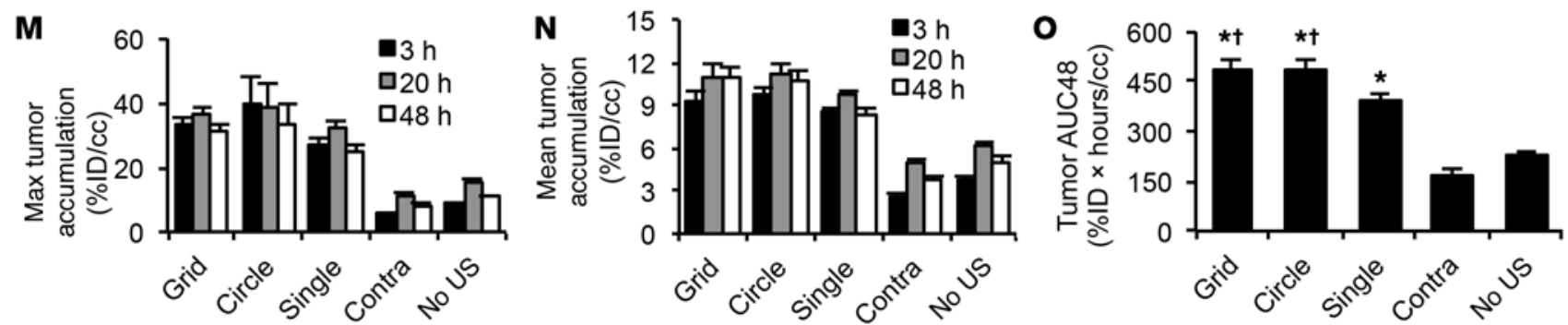

Figure 1. MRgFUS ablation enhanced accumulation of ${ }^{64} \mathbf{C u}-\mathbf{L C L}$ in NDL mammary carcinoma. (A) Three weeks after tumor implantation, tumors were ablated and imaged with pre- and postablation T1w-MRI. Mice were then injected with i.v. ${ }^{64} \mathrm{Cu}-\mathrm{LCL}$ and imaged with PET-CT at 3, 20, and 48 hours after treatment. Control treatment was no ultrasound (no US). (B-J) MRgFUS protocols and temperature profiles. T1w images localize ultrasound for (B) singlepoint, (C) circle, and (D) grid protocols. MR thermometry images following (E) 20 seconds single-point or (F) 60 seconds circle protocol MRgFUS. Temperature of measured and simulated ultrasound focus is plotted after (G) 20 seconds single-point or (H) 60 seconds circle protocol. Temperature distribution was simulated following (I) 20 seconds single-point or (J) 60 seconds circle protocol. Grid protocol shown in $\mathbf{D}$ and $\mathbf{M}-\mathbf{O}$ is equivalent to $\mathbf{E}$ and $\mathbf{G}$ repeated in grid pattern, due to sequential application of ablation points and cool-down period. (K) PET-CT maximum intensity projection images of ${ }^{64} \mathrm{Cu}$-LCL without ablation. White arrows indicate tumors; yellow arrowheads indicate jugular vein (JV), heart (H), and liver (L). (L) At 3, 20 , and 48 hours following singlepoint ablation (blue arrows), liposome accumulation is enhanced as compared with that in contralateral tumors (white arrows) in $\mathrm{PET}$-CT images. ${ }^{64} \mathrm{Cu}-\mathrm{LCL}$ PET image-derived volume of interest analysis plotted as $(\mathbf{M})$ maximum voxel intensity, $(\mathbf{N})$ average activity, and (0) AUC $\mathbf{C}_{48}$. Significance is analyzed with 1-way ANOVA followed by Tukey's test. For $\mathbf{M}-\mathbf{0}, n=4,3,12,19$, and 6 mice for grid, circle, single point, contralateral tumors (contra), and no treatment. Scale bars: $1 \mathrm{~cm}$. Thermometry color bars range from $70^{\circ} \mathrm{C}$ to $40^{\circ} \mathrm{C}$; PET color bar ranges from $25 \%$ ID/cc to $0 \%$ ID/cc. ${ }^{*} P<1 \times 10^{-4}$ vs. untreated tumors; ${ }^{\dagger} P<0.05$ vs. single-point ablation. Data are pooled from 5 experiments.

hours after ablation, the radioactivity in the heart, liver, and jugular veins decreased and radioactivity associated with the ablated tumors increased relative to the vascular and tissue background (Figure 1L). Radioactivity associated with clearance organs (gut and bladder) was also increased at later time points (Figure $1, \mathrm{~K}$ and L). At 48 hours, whole-body radioactivity had decreased, although activity within and surrounding the ablated tumor remained comparable to that observed at 20 hours (Figure 1L).

With all MRgFUS protocols, at 3, 20, and 48 hours after ablation, the mean and maximum accumulation greatly increased in 


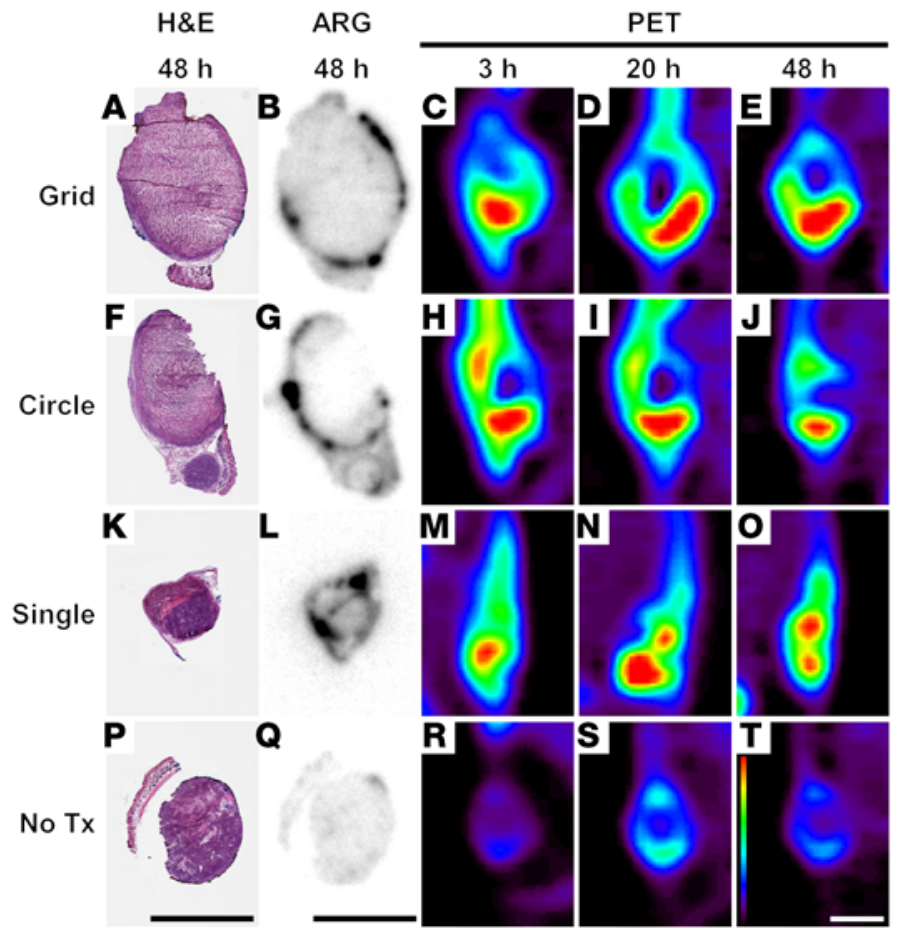

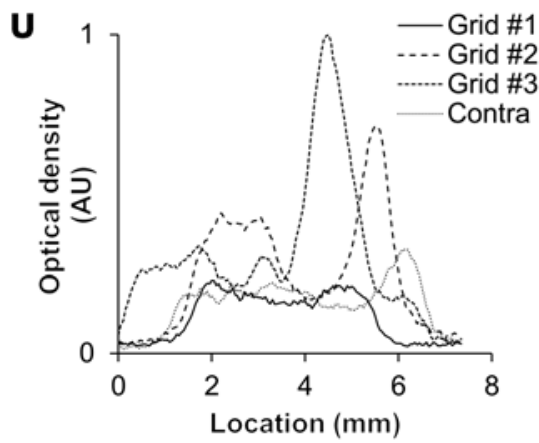

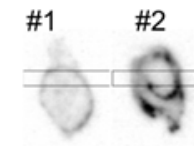

\#3 Contra
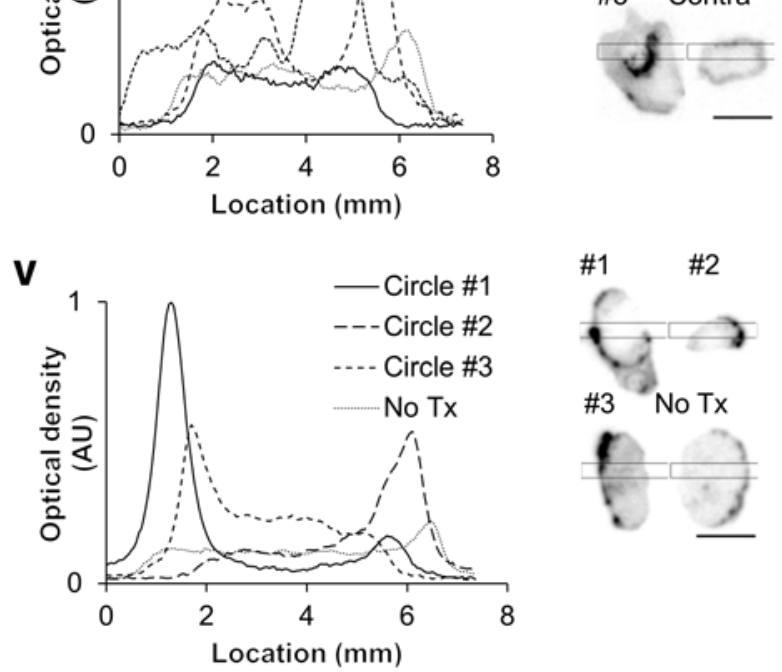

Figure 2. Ablation greatly enhances ${ }^{64} \mathrm{Cu}-\mathrm{LCL}$ accumulation in tumor rim and remaining viable tumor in the NDL tumor model. (A-T) Comparison of H\&E-stained frozen sections (A, F, K, and $\mathbf{P})$, autoradiography (B, G, L, and Q), and PET image slices (C-E, H-J, M-0, R-T) acquired at 3 hours, 20 hours, and 48 hours from tumors sectioned 48 hours after MRgFUS treatment with grid, circle, single-point ablation, or no ultrasound. Grid and circle MRgFUS exposures increased accumulation of ${ }^{64} \mathrm{Cu}-\mathrm{LCL}$ in rims surrounding ablation (dark purple rim on $\mathbf{A}$ and $\mathbf{F}$ corresponds with black rim on $\mathbf{B}$ and $\mathbf{G}$ ). Limited resolution of PET images blurs rim in $\mathbf{C}-\mathbf{E}$ and $\mathbf{H}-\mathbf{J}$, but also shows blood and lymphatic radioactivity in $\mathbf{C}-\mathbf{E}, \mathbf{H}-\mathbf{J}$, and $\mathbf{M}-\mathbf{O}$. Single-point ablation also increased accumulation of liposomes (L-0). Accumulation in untreated tumor was homogeneous and low (Q-T). (U and $\mathbf{V})$ Region of interest analysis of autoradiography (rectangular black box was plotted as line profile) demonstrating that central accumulation in tumors treated with (U) grid protocol and (V) circle protocol is comparable to that in untreated tumors in 5 of 6 tumors. $n=4,3,12,19$, and 6 mice for grid, circle, single point, contralateral tumors, and no treatment. Scale bars: $5 \mathrm{~mm}$. Color bar scaled from 25\%ID/cc to 0\%ID/cc. Data are representative of 5 experiments.

the insonified tumor and peritumoral region (blue arrows, Figure $1 \mathrm{~L})$ compared with that in the contralateral controls. With the exception of the insonified tumor, a similar volume of distribution was observed in the untreated and insonified mice (Figure 1, K and L), and accumulation in the contralateral tumor was not significantly changed from that of the untreated mice at any time point (white arrows, Figure 1, K and L). Additional PET images from the study are provided in Supplemental Figure 2.

Quantification of the accumulation verified that MRgFUS enhanced accumulation of ${ }^{64} \mathrm{Cu}-\mathrm{LCL}$ as compared with both untreated and contralateral controls (Figure 1, M and N). Enhanced tumor accumulation from MRgFUS ablation primarily occurred less than 3 hours after insonation and persisted through 48 hours, with more than $80 \%$ of the highest observed accumulation present at 3 hours after insonation. The increase in the spatial maximum accumulation in the treated versus control tumors was larger than the change in the mean accumulation, as might be expected for a local therapy (Figure 1, M and N). The greatest spatial maximum accumulation was observed at 3 hours after insonation, with accumulation of $39.7 \pm 8.6,33.8 \pm 1.9,27.4 \pm 2.1$, and $8.6 \pm 0.8$ percent injected dose per cc of tissue (\%ID/cc, mean \pm SEM) in circle, grid, singlepoint protocols, and untreated tumors, respectively (Figure $1 \mathrm{M}$ ). The spatial mean accumulation at 3 hours was $9.8 \pm 0.5,9.2 \pm 0.7,8.5$ \pm 0.4 , and $3.8 \pm 0.3 \% \mathrm{ID} / \mathrm{cc}$ in circle, grid, single-point protocols, and untreated tumors, respectively (Figure $1 \mathrm{~N}$ ). Further, the mean accumulation at 20 and 48 hours was similar to that in the earlier time points. Clearance in the clinically relevant circle and grid protocols was small; the decrease from the peak to the 48-hour accumulation was $5.4 \%, 1.8 \%, 13.6 \%$, and $17.2 \%$ in the circle, grid, single-point protocols, and no-treatment groups, respectively.

Based on the 48-hour PET data, the AUC over 48 hours $\left(\mathrm{AUC}_{48}\right)$ for tumors treated with grid, circle, and single-point protocols of MRgFUS was significantly greater than that for untreated tumors $\left(P=1.2 \times 10^{-5}, P=1.25 \times 10^{-5}, P=1.54 \times 10^{-5}\right)$. Further, the $\mathrm{AUC}_{48}$ for grid and circle application of MRgFUS was significantly greater than that achieved with the single-point ablation $(P$ $=0.025$ and 0.041), while the $\mathrm{AUC}_{48}$ for grid and circle protocols did not significantly differ (Figure 1O) and did not correlate with tumor size (Supplemental Figure 1E). A linear regression of tumor volumes and tumor accumulation at 3 hours demonstrated a slope of $-0.0074 \% \mathrm{ID} / \mathrm{cc}$ tumor accumulation per $0.01 \mathrm{cc}$ tumor volume with a Pearson's $R^{2}$ of 0.14 and with the smallest and largest tumors measuring $0.0087 \mathrm{cc}$ (4 mm longest diameter) and $0.10 \mathrm{cc}$ (6 mm longest diameter), respectively (Supplemental Figure 1E).

Biodistribution validates the macroscopic imaging finding of enhanced accumulation. Biodistribution at 48 hours confirmed that the effect of MRgFUS ablation on ${ }^{64} \mathrm{Cu}-\mathrm{LCL}$ distribution was confined to the tumor (Supplemental Figure 3A). Accumulation was 
A
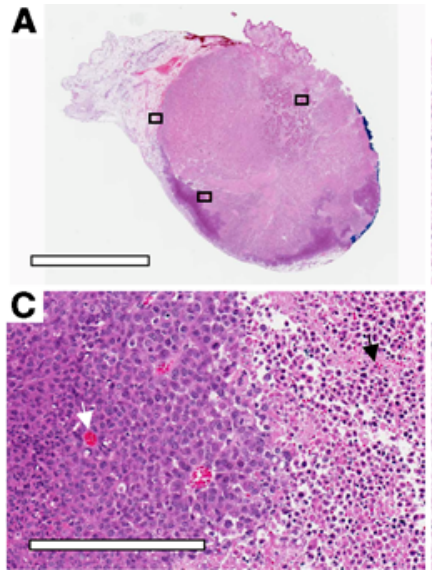

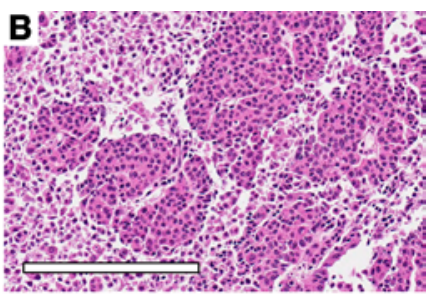

D.

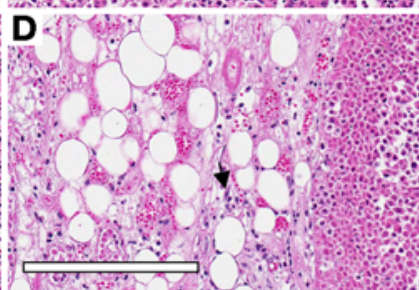

Figure 3. Mechanisms for enhanced accumulation after ablation at the boundary between viable and nonviable tissue stained with H\&E in the NDL tumor model. (A) Representative tumor following circle protocol ablation with orientation dye at boundary and black boxes at locations of insets. (B) View of heat-fixed tissue central to beam with surrounding loss of cell-cell adhesion. Necrotic tissue displays loss of glandular tumor architecture visible in heat-fixed and viable tumor. (C) View at border of viable (left) and necrotic (right) tumor and (D) view at border of mammary fat pad with light inflammatory reaction in stroma (black arrow in D) and necrotic tissue (right). (C and D) Viable fat pad and tumor tissue demonstrate dilated blood vessels (white arrow in $\mathbf{C}$ ) and extravasation of blood cells (black arrow in C), indicating disruption of vascular integrity. Histology was included in all $(n=117)$ studies. Scale bars: $4 \mathrm{~mm}$ (A); $200 \mu \mathrm{m}$ (B-D).

greater in treated compared with untreated tumors; however, as expected, the differences were smaller at 48 hours compared with the PET data corresponding to earlier time points (Figure 1N, Supplemental Figure $3 \mathrm{~A})$. Radioactivity was greatest $(>4 \% \mathrm{ID} / \mathrm{g}$ ) in the urine, liver, kidneys, spleen, and tumors of treated and untreated mice. Moderate activity (between 2 and $4 \% \mathrm{ID} / \mathrm{g}$ ) was observed in blood, heart, lung, and intestine, while very little activity $(<1 \% \mathrm{ID} / \mathrm{g})$ was observed in muscle, bone, and brain.

Accumulation within the ablated region and surrounding rim was visualized in the NDL model by microscopic scale imaging using histology and autoradiography. Microscopic examination of tumor sections revealed the extent of tumor damage produced by MRgFUS and the resulting local accumulation of radioactivity within remaining tumor (Figure 2). The bulk of the tumor was destroyed in tumors treated with grid and circle protocols, with small $(<1$ $\mathrm{mm}$ ) rims of viable tumor tissue remaining at the periphery of the tumor (Figure 2, A and F, and Supplemental Figure 4, B and F). Radioactivity was enhanced in these rims of viable tissue on autoradiography at 48 hours after treatment (Figure 2, B and G), which compares well to PET imaging (Figure 2, C-E and $\mathrm{H}-\mathrm{J}$ ). ${ }^{64} \mathrm{Cu}-\mathrm{LCL}$ accumulated in the rim of MRgFUS-treated tumors (Figure 2, $\mathrm{A}-\mathrm{O}$ ) to a greater degree than in untreated tumors (Figure 2, $\mathrm{P}-\mathrm{T}$ ). In order to compare the ${ }^{64} \mathrm{Cu}-\mathrm{LCL}$ accumulation in the tumor center and periphery between the control and treated tumors, we present a horizontal line drawn across the autoradiography images as a $2 \mathrm{D}$ line plot (Figure 2, $\mathrm{U}$ and V, line plots and corresponding images). In the tumor periphery, we found that the accumulation was increased in all circle protocol tumors. Accumulation in the ablated tumor center was comparable to that observed in contra-

Figure 4. As compared with controls, grid and circular ablation protocols alter the tumor distribution of a single injection of gadoteridol contrast agent in the NDL tumor model. Results were similar whether gadoteridol was injected before or after ablation. Columns represent the same tumor imaged at 5 minutes (A-E) and 3 hours (F-J) after ablation with the following protocols: ( $\mathbf{A}$ and $\mathbf{F})$ grid, (B and $\mathbf{G})$ circle, (C and $\mathbf{H})$ single point, ( $\mathbf{D}$ and $\mathbf{I})$ no ultrasound, gadoteridol before ablation, (E and $\mathbf{J}$ ) circle ablation with no gadoteridol. (K) For gadoteridol injection after ablation, ratio of intensity in ablation region and quadriceps muscle reaches $3.2 \pm 0.4$ at 1.5 hours, $n=4$. (A-J) Representative images with $n=4$ each for grid, single-point ablation, no ultrasound plus gadoteridol (Gd), and circle no gadoteridol, $n=12$ for circle plus gadoteridol. Scale bar: $5 \mathrm{~mm}$. Data are pooled from 2 experiments. lateral and untreated tumors (5 of 6 ablated tumors in Figure 2, $\mathrm{U}$ and $\mathrm{V}$, had equal to or greater accumulation, which is representative of the study).

Viability staining confirmed the creation of a large nonviable region by MRgFUS ablation, although small rims of viable tissue were found 24 hours after MRgFUS treatment (Supplemental Figure 4). The enhanced accumulation of a fluorescent dye that was sequestered in the core of the liposomes within the tumor rim further validated the enhancement resulting from ablation (Supplemental Figure 4).

Histomorphology by H\&E demonstrated biologic response to MRgFUS, including adjacent tissue reaction (Figure 3). At the center of the treatment area, the tissue appeared heat fixed, with shrunken but intact nuclei, increased cytoplasmic eosinophilia, and some preservation of tissue architecture (Figure 3B). Immediately surrounding the treated area, the tissue was discohesive and nonviable, with ghosted and pyknotic nuclei (Figure 3B, left, and Figure 3C, right).
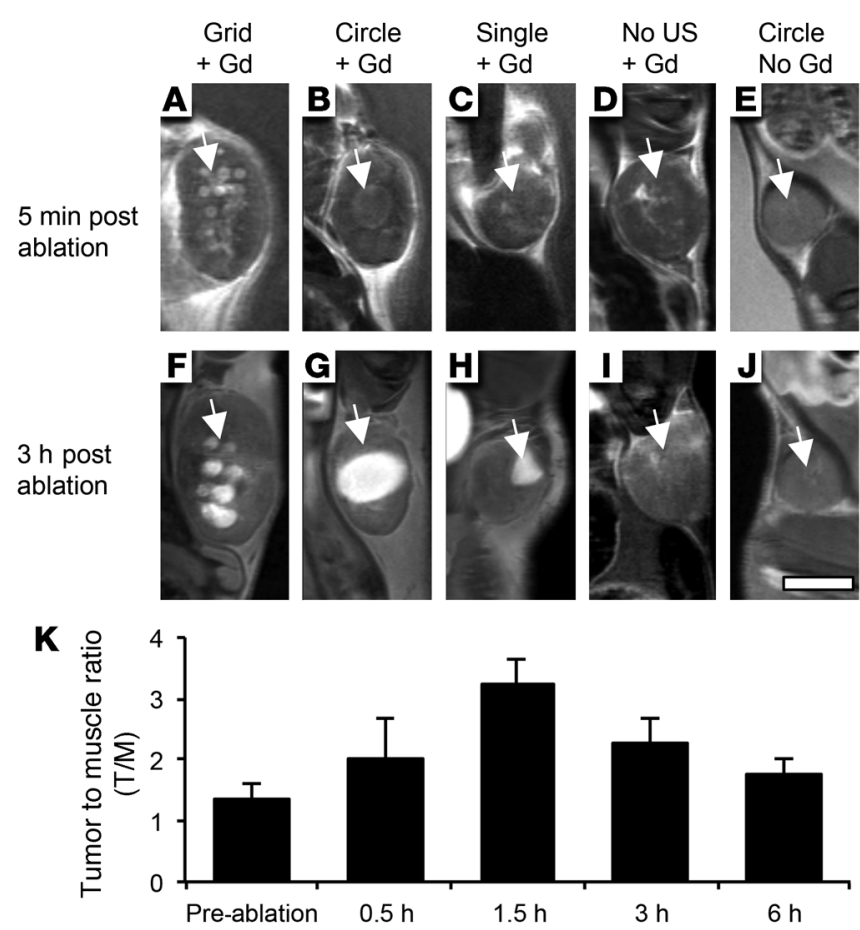
A

\begin{tabular}{|c|c|}
\hline 0 wk & Implant \\
\hline 3 and $4 \mathrm{wk}$ & $\begin{array}{l}\text { MRI } \\
\text { Ablation or control (no US) } \\
\text { Inject Dox-LCL i.v. }\end{array}$ \\
\hline $5 \mathrm{wk}$ & $\begin{array}{l}\text { MRI } \\
\text { Ablation or control (no US) } \\
\text { Inject }{ }^{64} \text { Cu-LCL i.v. }\end{array}$ \\
\hline $\begin{array}{r}5 \mathrm{wk}+ \\
3,20 \text {, and } 48 \mathrm{~h}\end{array}$ & $\begin{array}{l}\text { MRI } \\
\mathrm{PET} / \mathrm{CT}\end{array}$ \\
\hline $\begin{array}{r}5 w k+ \\
48 \mathrm{~h}\end{array}$ & Histology \& autoradiography \\
\hline
\end{tabular}

B

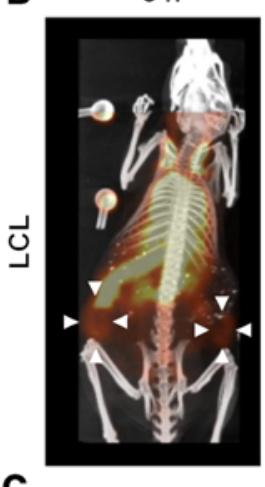

C

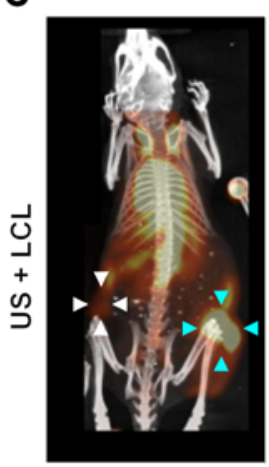

$20 \mathrm{~h}$
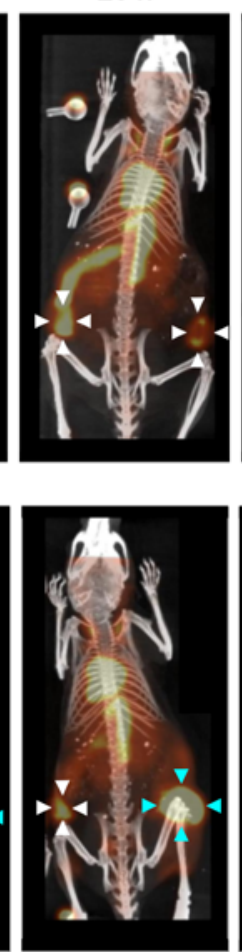

$48 \mathrm{~h}$
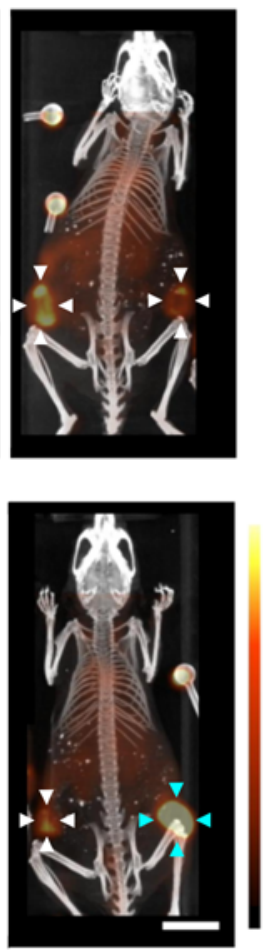

D

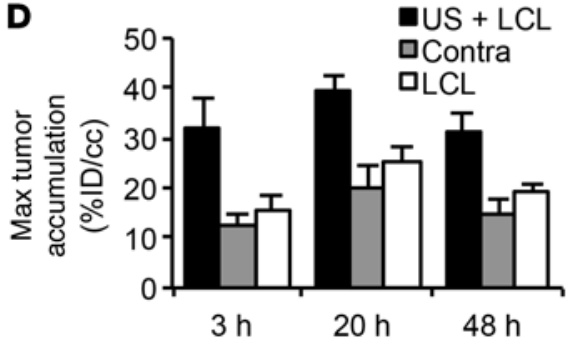

E

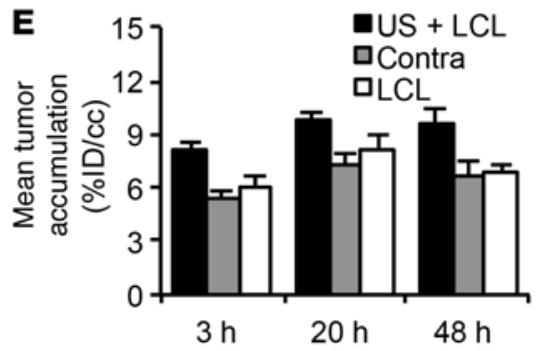

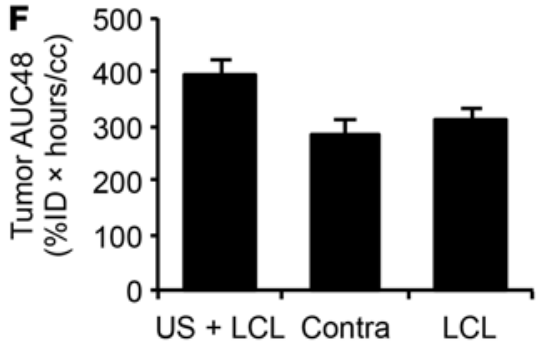

Figure 5. Single-point MRgFUS ablation continues to enhance tumor accumulation of liposomes in the NDL tumor model after treatment for $\mathbf{2}$ weeks with MRgFUS ablation and therapeutic doxorubicin liposomes (Dox-LCL). (A) Mice were treated with weekly ablation (single-point protocol) or control (no ultrasound) and biweekly Dox-LCL (i.v., $6 \mathrm{mg} / \mathrm{kg}$ ) for 2 weeks, then treated with ablation or control (no ultrasound) and injected with ${ }^{64} \mathrm{Cu}-\mathrm{LCL}$. (B) PET-CT MIP images of mice treated only with repeated Dox-LCL (LCL) demonstrate heterogeneous tumor accumulation of ${ }^{64} \mathrm{Cu}-\mathrm{LCL}$ (white arrows). (C) PET-CT MIP images of mice treated with ablation plus Dox-LCL (US + LCL) reveal enhanced accumulation in ultrasound-treated tumors (blue arrows). PET image-derived tumor activity of ${ }^{64} \mathrm{Cu}-\mathrm{LCL}$ was obtained by manual segmentation with data plotted as (D) maximum pixel intensity within volume of interest, (E) activity averaged over volume of interest, and (F) $\mathrm{AUC}_{48} \cdot n=3$ each for US $+\mathrm{LCL}$, contralateral tumors, and LCL. Scale bar: $1 \mathrm{~cm}$. PET color bar ranges from $25 \% \mathrm{ID} / \mathrm{cc}$ to $0 \% \mathrm{ID} / \mathrm{cc}$.

In some areas a viable rim of tumor was observed, with large blue nuclei, intact tumor structural integrity, and dilated blood vessels (Figure 3C, left). Necrotic tumor close to the border between viable and necrotic tissue included extravasated red blood cells (Figure 3C, right, and Figure 3D, right). Dilated vasculature and an inflammatory reaction was observed within the mammary fat pad bordering tumor (Figure 3D, left). Dilated vasculature, an inflammatory reaction, discohesive tissue, destruction of tissue architecture, and loss of vascular integrity resulting in extravasation of blood cells represent potential mechanisms for enhanced accumulation of nanoparticles.

Validation of the effect of ablation in a second syngeneic tumor model (4T1) with enhanced vascular permeability. In order to verify that the enhanced accumulation resulting from ablation is generally applicable to a range of tumor phenotypes, the study described above was also performed in the $4 \mathrm{~T} 1$ mouse model of syngeneic cancer (Supplemental Figures 5-7). While the NDL tumors had a predominantly epithelial phenotype, by morphology, 4T1 tumors demonstrated a mesenchymal-transition phenotype with highly proliferative spindle-shaped tumor cells that infiltrated the fat pad (Supplemental Figure 5A) and the abdominal wall muscle upon surgical excision. Following ablation, a region of necrosis was clearly visible, with a small surrounding region of viable tumor cells (Supplemental Figure 5B).

As expected, the accumulation of LCL was greatly increased in the region immediately surrounding the ablated site as early as 3 hours after ablation, as demonstrated by PET imaging, autoradiography, and PET volume of interest analysis (Supplemental Figures 6 and 7). Differences in the morphology of the necrosis were visible between the single-point and circle ablation protocols (Supplemental Figure 6C). In the $4 \mathrm{~T} 1$ tumor model, at 3 hours after insonation, 
A

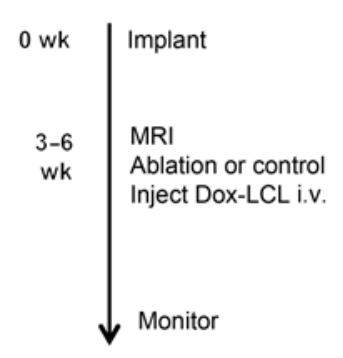

D

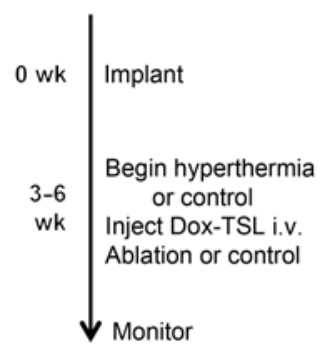

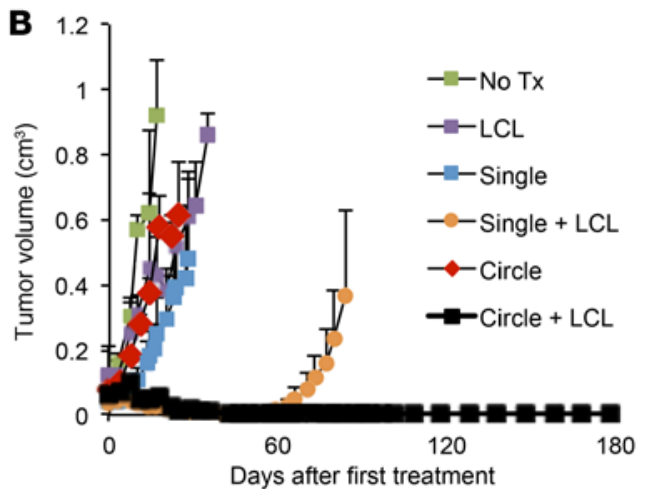

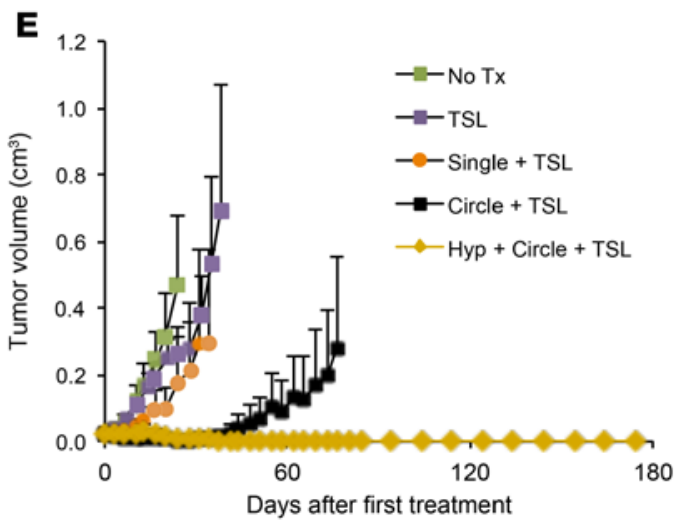

C

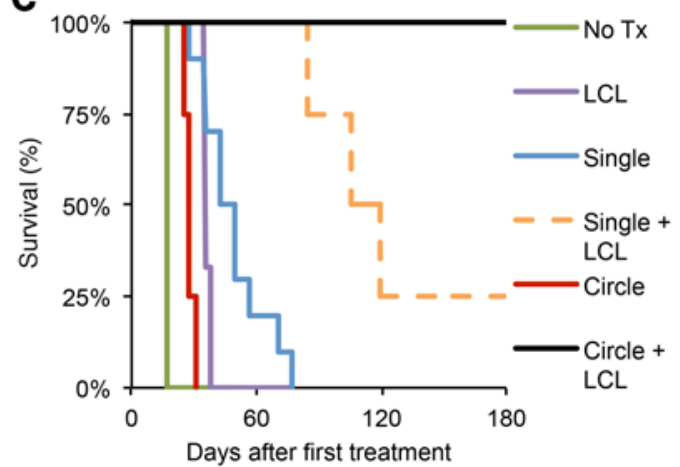

$\mathbf{F}$

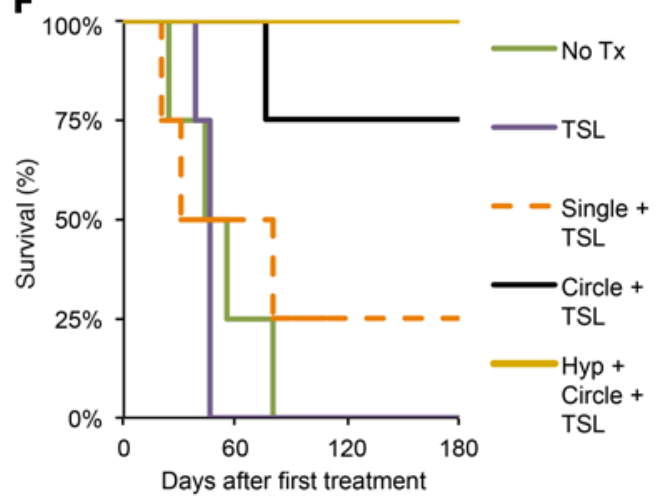

Figure 6. Combination of ablation and doxorubicin encapsulated in LCL (Dox-LCL) or TSL (Dox-TSL) can produce complete local regression in the NDL tumor model. (A-C) Treatments included weekly single-point ablation (single, $n=8$ ), circle pattern ablation (circle, $n=4$ ), single-point ablation plus Dox-LCL (single $+\mathrm{LCL}, n=4$ ), circle ablation plus Dox-LCL (circle $+\mathrm{LCL}, n=4$ ), no ultrasound and no drug (no tx, $n=8$ ), or Dox-LCL only (LCL, $n=4$ ). Dox-LCL (or control saline) was injected biweekly (4 ablations and 8 injections total). (B) At 180 days after the start of treatment/152 days after completion (study end point), tumors could not be detected in mice treated with circle ablation plus Dox-LCL. In all other groups, tumor growth was delayed, but a complete response was not typically achieved. (C) At study end point, all $(n=4)$ mice treated with circle ablation plus Dox-LCL and $25 \%(1$ of 4$)$ of mice treated with single-point ablation plus Dox-LCL survived. (D-F) TSL therapy combined with ablation. (D) Treatments included weekly: single-point ablation plus Dox-TSL (single + TSL, $n=4$ ), circle ablation plus Dox-TSL (circle $+\mathrm{TSL}, n=4$ ), circle ablation preceded by hyperthermia and Dox-TSL (hyp + circle + TSL, $n=4$ ), no ultrasound and no drug (no tx, $n=4)$, or Dox-TSL only $(n=4)$ for a period of 4 weeks. (E and F) At study end point, tumors could not be detected in all mice treated with circle ablation plus Dox-TSL combined with hyperthermia. In all other groups, tumor growth was delayed, and a complete response was also achieved in $75 \%$ of mice treated with circle ablation plus Dox-TSL and 25\% of mice treated with single-point ablation plus Dox-TSL. Data are pooled from 6 experiments.

the spatial maximum accumulation in the circle, single-point protocol, and untreated tumors was $58.7 \pm 5.16,53.5 \pm 3.10$, and 18.5 $\pm 2.60 \% \mathrm{ID} / \mathrm{cc}$ (Supplemental Figure 6D). Without treatment, the accumulation of nanoparticles was greater in the $4 \mathrm{~T} 1$ than in the NDL model (likely due to a greater EPR effect). The increased AUC remained significant in the $4 \mathrm{~T} 1$ model, and the mean and maximum accumulation achieved after ablation were larger in the 4T1 than in the NDL tumor model (Supplemental Figure 6D).

Accumulation of small molecule was enhanced on CET1wMRI. We found that gadoteridol provided a high-contrast image of the ablated regions. Following a single gadoteridol injection at the time of ablation (Figure 4), the pattern of ultrasound exposure was clearly visible at 3 hours after treatment with grid (Figure 4, A and F), circular (Figure 4, B and G), and single-point (Figure $4, \mathrm{C}$ and $\mathrm{H}$ ) protocols; here, these general results are shown in the NDL tumor model. The region of enhancement based on the small molecule accumulation was smaller than the region of ablated tissue assessed on histology after 48 hours. For example, Figure $4 \mathrm{G}$ is a typical image resulting from the circle ablation protocol; Figure $3 \mathrm{~A}$ represents the typical histological finding for the same protocol. No local enhancement was observed without ablation (Figure 4, D and I) or gadoteridol injection (Figure 4, E and J). The maximum observed tumor to muscle (T/M) ratio for gadoteridol injected following ablation was $3.2 \pm 0.4$; the peak occurred at 1.5 hours after ablation and fell to $1.8 \pm 0.3$ at 6 hours (Figure $4 \mathrm{~K}$ ). When gadoteridol was administered before ablation, we found that the peak T/M ratio was $3.4 \pm 0.2$ in the first hours after ablation and returned to baseline within hours. Thus, no significant difference between administration of gadoteridol before and after ablation was observed.

Enhancement of accumulation persisted over multiple treatments with chemotherapy. We then added multiple treatments with ablation combined with doxorubicin-containing nanoparticles to determine whether ablation would continue to enhance chemotherapy delivery (Figure 5A). Repeated chemotherapy treatment with MRgFUS and LCL-encapsulating doxorubicin (Dox-LCL) did not diminish the enhanced accumulation of ${ }^{64} \mathrm{Cu}$-LCL (Figure 5). NDL tumor mice treated with Dox-LCL alone for 2 weeks revealed enhanced splenic activity as well as heterogeneous tumor accumulation of ${ }^{64} \mathrm{Cu}-\mathrm{LCL}$ (white arrows, Figure 5B). How- 
ever, enhanced accumulation of ${ }^{64} \mathrm{Cu}-\mathrm{LCL}$ in treated tumors was observed even in mice treated for 2 weeks with both Dox-LCL and single-point MRgFUS (blue arrows, Figure 5C). Given that ablation was applied only in a single point within the center of the tumor, the maximum accumulation can best quantify the effect. In this cadre, the spatial maximum of tumor accumulation was enhanced at all time points, with accumulation of $31.6 \pm 6.2 \% \mathrm{ID} /$ cc and $15.7 \pm 2.8 \% \mathrm{ID} / \mathrm{cc}$ in repeated MRgFUS-treated and control tumors, respectively, at 3 hours after insonation and injection (Figure 5D). The mean tumor accumulation was also enhanced at all time points, with accumulation of $8.2 \pm 0.4 \% \mathrm{ID} / \mathrm{cc}$ and $6.0 \pm$ $0.6 \% \mathrm{ID} / \mathrm{cc}$ in repeatedly treated and control tumors, respectively, at 3 hours (Figure 5E). The $\mathrm{AUC}_{48}$ for the repeated combined treatment was greater than the contralateral and drug-only treatment, but the result was not significant, as repeated treatments appeared to modestly enhance accumulation in tumors not treated with ultrasound (Figure $5 \mathrm{~F}$ ).

MRgFUS and Dox-LCL lengthened survival. Since the circular ablation protocol produced the greatest Dox-LCL accumulation, survival was compared with this and other control protocols in the NDL tumor model (Figure 6A). With the combination of circle ablation and Dox-LCL, tumors were eliminated in all mice and recurrence was not observed within 180 days (Figure 6B); the combination of circle ablation and Dox-LCL was the most effective in enhancing survival (Figure 6C). Median survival times for untreated, single-point ablation, circle-pattern ablation, and LCL protocols were all below 50 days, as compared with untreated tumors, which resulted in survival of less than 17 days (Figure 6C). The combination of MRgFUS, Dox-LCL, and single-point ablation increased survival to $111 \pm 19$ days, with recurrence detected in 3 of 4 mice after approximately 120 days and no recurrence detected in the remaining mouse after 180 days.

Dox-TSL combined with ablation also reduced tumor growth and achieved a complete response in the NDL model. Liposomes can also be formulated to release the drug in response to increased temperature, although at a cost of decreased circulation stability. Therefore, we evaluated whether treatment with the thermally sensitive doxorubicin liposomal formulations previously developed by our group (12) were also curative using single-point or circle ablation (Figure 6, D-F). The synthesis and components were similar to the LCL used in all other studies, with the exception of the inclusion of lipids with a lower phase transition temperature; therefore, the results can be directly compared. As ablation protocols increase the tissue temperature for a very short time, the addition of a period of sustained hyperthermia was also evaluated in this survival study (Figure 6D). In order to release the drug at the site, the liposomes were injected before ablation.

Tumor growth was greatly reduced by combining circle ablation with TSL (Figure 6E); however, without the inclusion of hyperthermia, regrowth after the end of treatment was observed in 1 of 4 mice. We found that protocols combining TSL with ablation using the single-point and circle protocols were curative in a fraction of mice ( $25 \%$ and $75 \%$, respectively). When the ablative therapy was preceded by a 25 -minute application of hyperthermia designed to release the drug from the particle, $100 \%$ of the mice (4 of 4) achieved a complete response with TSL combined with the circle ablation protocol and tumors did not recur within 180 days.

\section{Discussion}

As compared with other ablative techniques, MRgFUS provides an improved opportunity for image guidance of conformal therapy, as the temperature, thermal dose, and beam location can each be assessed. Yet adequate treatment of the tumor margins can still be challenging near sensitive structures, such as nerves and blood vessels. Therefore, the combination of conformal ablation with chemotherapy has the potential to enhance local and systemic efficacy. In the preclinical orthotopic and immunocompetent models studied here, nearby organs and skin limit the ablated region and provide the opportunity to test clinically relevant protocols.

Our primary goal in this paper was to examine the mechanisms and ultimate outcome achieved by combining long-circulating liposomal therapy with ultrasound ablation. Most importantly, we found that the circular ablation protocol leaving a thin $(200 \mu \mathrm{m})$ rim of viable tissue was locally curative when combined with liposomal therapy. When the remaining rim was larger in diameter (as with the single-point ablation), the combined treatment extended survival, but in most cases, tumor growth returned (Supplemental Figure 1B). While we focused on the use of the circle ablation protocol for the survival study due to the relative speed and ease of administration, it is likely that similar results could be obtained with the grid ablation protocol. The accumulation of drug was similar between the circle and grid protocols, and a similar elimination of much of the viable tumor could be achieved with the grid protocol. However, the periablative region instantaneously exposed to hyperthermia was larger, and enhanced accumulation was comparable between the rapid circular and slower grid ablation protocols. Therefore, the combination of the circle protocol and nanoformulations may be advantageous for enhanced delivery of nanotherapies with a short circulation time.

Temperature-sensitive formulations are increasingly under consideration for inclusion in combination protocols involving ultrasound hyperthermia and ablation in clinical care (39). The advantage of such nanoparticles is that the drug is rapidly released from the particle by heat and therefore becomes available to both the endothelial and tumor cells. The disadvantage of this approach is that the particles typically have a shorter circulation half-life as compared with other nanoformulations. We have previously shown that a highly effective formulation of temperature-sensitive doxorubicin particles could be synthesized with a 1.5-hour circulation half-life (as compared with an 20 hour circulation half-life for long-circulating particles) $(12,40)$. We showed that the free drug was released only in the presence of heat and reduced $\mathrm{pH}$ and therefore systemic toxicity was reduced. Due to the relatively short systemic circulation, release of the drug from temperature-sensitive formulation at the tumor site is required for treatment efficacy, as it greatly increases accumulation. Here, we confirmed that an effective therapy could be achieved by combining ablation with a temperature-sensitive formulation; however, this treatment protocol was fully curative in the entire cohort only when adding a brief period ( 25 minutes) of hyperthermia to the protocol. When hyperthermia was used to release the drug prior to ablation, tumors were eliminated in $100 \%$ of the treated mice within 4 treatments. Although hyperthermia combined with TSL can be curative for local disease, the addition of ablation is attractive for poorly perfused tumors, as the released small molecule 
will diffuse and accumulate, highly necrotic tumor can be directly ablated, and ablation will further increase the accumulation of particles and drug within the region.

While ultrasound-mediated hyperthermia alone also enhances the accumulation of LCL in and around tumors, we have previously demonstrated that the optimal settings for using hyperthermia to enhance nanoparticle accumulation are dependent on the tumor biology (21). While low temperatures and pressures increase LCL accumulation in epithelial tumors, higher temperature can result in vascular stasis. Alternatively, higher temperatures and pressures are required for treatment of epithelial-mesenchymal transition (EMT) tumors (21). Further, the improvement in the accumulation of LCL with mild hyperthermia was on the order of 1.5-fold, which is substantially less than that achieved with ablation here. In addition, ablation can directly reduce the tumor mass and thus synergize with the drug therapy. Therefore, taken together, our studies indicate that the 2 protocols developed here with circle ablation and nanotherapy (circle ablation with LCL and circle ablation with hyperthermia and TSL) are both superior candidates for the treatment of tumors bordering on sensitive structures.

Distribution and mechanisms for combining ablation with liposomal therapy. As a result of the combination of ablation and long-circulation liposomal therapy, nanoparticle accumulation was greatest within nearby vasculature and the thin rim (approximately 200-300 $\mu \mathrm{m})$ of viable tumor immediately surrounding the treated region, as demonstrated by H\&E, autoradiography, fluorescence imaging, and viability staining (Figure 2 and Supplemental Figure 4). Mechanisms for enhanced nanoparticle delivery included engorged and leaky vasculature and a local inflammatory response, as illustrated in Figure 3. Regarding the mechanism for the gadoteridol accumulation in the center of the ablated region, we hypothesize that edema immediately following ablation is responsible for carrying and concentrating this small molecule within the damaged tissue.

Accumulation of model drug and drug carriers. All ablation protocols evaluated here enhanced the accumulation of ${ }^{64} \mathrm{Cu}-\mathrm{LCL}$ within tumors, with approximately $80 \%$ of the accumulation occurring within 3 hours after ablation and less than $10 \%$ cleared within 48 hours using the clinically relevant grid and circle protocols. Further, gadoteridol accumulated in the ablated region within 1.5 hours after ablation, providing a clear image of the central ablation focus.

Quantification of enhanced accumulation confirmed the potential significance of pairing ablation and nanodelivery. In the 4T1 mouse model, administration of free doxorubicin without ablation resulted in tumor accumulation on the order of $1 \% \mathrm{ID} / \mathrm{cc}$ (41); nanoparticle formulations improved this by increasing circulation time and facilitating accumulation only in regions with leaky vasculature. The NDL orthotopic mammary tumor has a significantly lower native EPR effect than that the 4T1 (41); in our hands, the mean accumulation in NDL tumors has averaged 5\%-6\%ID/ $\mathrm{cc}$ in many studies. Ablation further increased nanoparticle accumulation. At 3 hours after insonation, the spatial maximum accumulation of nanoparticles in the NDL tumors was $39.7 \pm 8.6,33.8$ $\pm 1.9,27.4 \pm 2.1$, and $8.6 \pm 0.8 \% \mathrm{ID} / \mathrm{cc}$ in circle, grid, single-point protocols, and untreated tumors, respectively, and therefore the local accumulation of doxorubicin could be greatly increased. Similarly, in the $4 \mathrm{~T} 1$ tumor model, at 3 hours after insonation, the spatial maximum accumulation in the circle, single-point proto- col, and untreated tumors was $58.7 \pm 5.15,53.5 \pm 3.10$, and $18.5 \pm$ $2.60 \% \mathrm{ID} / \mathrm{cc}$. Taken together, as compared with the administration of free drug in the $4 \mathrm{~T} 1$ model, the results indicate that drug accumulation within regions of viable tumor near the ablated zone can be enhanced on the order of 50-fold by coupling nanoparticles and ablation.

As the resolution of PET is on the order of $1 \mathrm{~mm}$ and autoradiography is not fully quantitative, the peak accumulation within this thin rim may exceed the PET estimates. Therefore, the therapeutic impact of this enhancement within the remaining tumor rim may be even more significant than the macroscale PET accumulation measures would predict.

While the spatial maximum accumulation increased greatly, the tumor mean accumulation also increased, e.g., a single "grain of rice size" point of ablation increased the mean accumulation within an approximately $5-\mathrm{mm}$ tumor. This is likely due both to the direct effect of ablation on tumor vessels and to diffusion of heat resulting in mild hyperthermia in the remainder of the tumor. We questioned whether the application of the grid or circular protocols would reduce drug delivery within the ablated region as compared with that of control (untreated) tumors and regions. We found that in most cases, the LCL accumulation at 48 hours and gadoteridol accumulation at 3 hours were not reduced within the ablated tissue (Figure 2, U and V, and Figure 4). This likely results from transport from injured vessels to the lesion in the early time points after ablation.

Enhancement persists through repeated administration of $M R g-$ FUS ablation and Dox-LCL. Previous studies of multiple exposures to MRgFUS have been limited. Here, we demonstrated that ultrasonic enhancement of nanoparticle accumulation persisted following repeated administration of MRgFUS ablation and DoxLCL in the NDL model. This finding supports combining ablation with nanoparticle chemotherapy in palliation and the treatment of locally advanced disease. However, much work remains to be done to develop an optimal dosing strategy for this combination therapy.

Accumulation of the small molecule peaked within 1.5 hours; $80 \%$ of nanoparticle accumulation occurred within 3 hours. ${ }^{64} \mathrm{Cu}-\mathrm{LCL}$ PET tracers are a highly sensitive and quantitative method for tracking nanoparticles and provide the opportunity to track the kinetics of accumulation. Advances in PET/MR point to the clinical potential of ${ }^{64} \mathrm{Cu}-\mathrm{LCL}$ in conjunction with nanoparticle chemotherapy for therapeutic guidance, dosimetry, and assessment (42). The regional accumulation observed for both the liposomes and gadoteridol occurs rapidly, with the greatest changes within the first 3 hours after treatment. These observations support the concept of an enhanced drug delivery time window immediately after thermal ablation and may be important in choice of adjuvant chemotherapy, delivery vehicle, and dosing regimen. The rapid accumulation of the nanoparticle is important for 2 reasons: the tumor drug exposure over time is increased, and the tumor accumulation of targeted nanoparticles (which often have a short circulation half-life) will be enhanced. Based on the gadoteridol results, we further postulate that a targeted small molecule would accumulate after ablation and the targeting moiety could prevent subsequent clearance.

Strategies for enhanced drug delivery using thermal therapy are in clinical trials; for example, strategies incorporating TSL are being 
evaluated in breast cancer. This MRgFUS-triggered accumulation of small molecule gadolinium contrast may be useful for early therapeutic assessment. Further, preclinical work in our laboratory and others has indicated that a complete response can be obtained with a small number of treatments, even in aggressive cancers (12). MRgFUS provides a well-controlled environment to guide such therapy and the opportunity for conformal treatment; however, the logistics for the incorporation of such strategies in clinical practice are challenging. In most centers, facilities for treatment with ablation or hyperthermia are not colocated with infusion clinics. Optimal treatment results will be obtained with infusions administered prior to or at the time of thermal therapy, and therefore, optimization of clinical logistics will be important.

Protocols. We included 3 MRgFUS ablation protocols with a goal of comparing model therapeutic accumulation within and surrounding the ablated region. The accumulation was particularly intense for the circular and grid protocols and was least for the single-point ablation. A circular MRgFUS protocol has been shown to be a useful strategy for rapid delivery of high-intensity focused ultrasound to large tissue volumes (7). Continuous application of ultrasound to create a scanned circular geometry can save treatment time, but necessitates careful planning and thermal monitoring, particularly when treating larger tumor volumes (43). Here, we observe that the circular ablation protocol was capable of coagulating a large region of tumor, with a rapid, 60-second treatment time and, when augmented with chemotherapy, was curative. Alternatively, the application of multiple treatment points in a grid is applied in the ExAblate clinical system $(44,45)$ and from phased treatment arrays (46). Sequential treatment of multiple points requires rest periods for tissue cooling as well as frequent mechanical repositioning of the ultrasound transducer; therefore, we observed that a circular protocol provides substantial time savings in our model.

We predicted that the diameter of the spherical region of cell death would be approximately $5 \mathrm{~mm}$ with the circular ablation protocol (Figure 1G). We found that the region of cell death was similar to or slightly larger than expected (Figure $2 \mathrm{~F}$ and Supplemental Figure 4, F and $\mathrm{G}$ ). The smaller volume ablated with the singlepoint treatment also corresponded with the predicted dimensions.

We do not anticipate that replicating the results obtained here with a $3-\mathrm{MHz}$ center frequency will be problematic for organ systems that are currently accessible to ultrasound ablation. Highintensity focused ultrasound systems intended for treatment of uterine fibroids operate near $1 \mathrm{MHz}$, balancing thermal absorption and the size of the focal region with the need to penetrate deeply into the abdomen; however, ultrasound frequencies ranging from 0.8 to $10 \mathrm{MHz}$ have been used for ultrasound thermal therapy (47) and $3 \mathrm{MHz}$ transducers have been used for prostate cancer therapy, as in the Sonoblate 500 (48). In this study, we employed a 3-MHz transducer due to the smaller focal volume obtained as compared with a lower frequency. This was desirable to facilitate comparison of single-spot, grid, and circle ablation protocols in addition to avoiding gross heating of the mouse, severe skin burns, and insonation of the mouse intestine. Our similar results using grid and circle protocols (which do not have an equal rate of heating) suggest that enhancement of nanoparticle chemotherapy is not dependent upon the rate of heating. The effect of ultrasound on biological tis- sue is characterized by the thermal and mechanical effects, and the use of a higher frequency in this study ensures that mechanical effects of ultrasound are unlikely to contribute to the therapeutic effect achieved here. We used 3.1 MPa peak negative pressure (PNP) in this study; another recent study using $1.5 \mathrm{MHz}$ demonstrated that the cavitation threshold at that frequency was greater than 11 $\mathrm{MPa}$ (49). Therefore, if the frequency is reduced in ultimate clinical translation, the acoustic pressure can be greatly increased if needed to achieve the thermal dose used here without fear of cavitation. Further, a relatively low acoustic power and short insonation were sufficient to achieve the enhanced delivery. Thus, achieving similar results in future human studies should be feasible.

Comparison with previous work. Enhancement of nanoparticle accumulation in thermally ablated tumors has previously been demonstrated with minimally invasive RF thermal ablation (4-6). Previous work with Doxil and RF ablation in a canine sarcoma model suggested that accumulation is dramatically enhanced within a peripheral ring of hyperthermia about the central ablated region (4). Using MRgFUS, a noninvasive and conformal method of ablative thermal therapy, we found that enhancement is not dependent upon physical damage due to the introduction of a catheter or RF probe. The circular trajectory applied here is likely to most closely approximate $\mathrm{RF}$ ablation, since a larger $(5 \mathrm{~mm}$ ) region is heated instantaneously. The circulation time achieved with the liposomes used here (with or without ablation) was similar to that observed for Doxil in BALB/c mice with implanted $4 \mathrm{~T} 1$ tumors without thermal ablation (41). Although all preclinical models differ in the EPR effect observed, here the peak accumulation of liposomes was greater following ultrasound ablation than that reported with Doxil (without ablation) (41).

Application of multiple heat treatments without regard to lifetime radiation limitations is one potential advantage of thermal ablative therapies. However, previous studies of RF thermal ablation in combination with LCL have examined a single application of heat and liposomes (36). Moreover, previous studies using MRgFUS in combination with liposomes have primarily focused on enhanced delivery following hyperthermia (50) as opposed to ablation. Here, we demonstrate that with noninvasive thermal ablation by MRgFUS, multiple applications of heat and liposomes are practical and can lead to durable complete response.

Study limitations. A limitation of this study is the assumption that the biodistribution of chemotherapeutic liposomes is the same as that of ${ }^{64} \mathrm{Cu}-\mathrm{LCL}$. We found that delivery was much more rapid after ablation, and the presence of drug was unlikely to alter the effect of ablation. In addition, small differences could be observed between the multiple methods used to image and quantify nanoparticle accumulation. These can be attributed to partial volume effects in PET that limit spatial accuracy, to the changes in tissue properties as a result of dissection and fixation, and to the challenges of coregistration across in vivo and ex vivo imaging methods.

In summary, PET-based quantification of the enhanced accumulation confirmed the potential significance of pairing ablation and nanodelivery, achieving high nanoparticle concentrations within a remaining viable rim. Further, MR-based methods illustrated the accumulation of a small molecule probe within the ablated tumor center. Potential mechanisms for the enhanced delivery include leaky and engorged vasculature and an inflam- 
matory response. The enhanced accumulation resulting from ablation persisted following repeated treatment with MRgFUS ablation and Dox-LCL. Moreover, we observe that for tumors with an initial diameter of approximately $4 \mathrm{~mm}$, the combination of doxorubicin-containing liposomes with a single point of thermal ablation was sufficient to extend survival, confirming the finding that treatment of a minimal tumor volume enhanced nanoparticle accumulation within the entire tumor. Expanding the ablated region such that only a thin rim of viable tumor remained and adding doxorubicin-containing liposomes eliminated the local disease in treated animals. These results suggest that MRgFUS and doxorubicin liposomes (long circulating or temperature sensitive) may be successful in palliation, rescue therapy, or debulking of tumors that are otherwise unresectable due to proximity to vital structures or tumor volume.

\section{Methods}

Preparation of $L C L$. Lipids and a miniextruder were obtained from Avanti Polar Lipids Inc.; other reagents were obtained from SigmaAldrich unless otherwise indicated. Liposomes used here included ${ }^{64} \mathrm{Cu}$-labeled empty LCL, doxorubicin-loaded LCL and TSL, and Alexa Fluor 555-loaded (A555-loaded) LCL. Dox-LCL was prepared using copper for stabilization, a formulation that reduces systemic toxicity by encapsulating nonbioactive copper-doxorubicin crystals (40) that dissociate in the low $\mathrm{pH}$ of the tumor microenvironment $(40,51)$. Copper-doxorubicin TSLs (Dox-TSL) demonstrate increased circulating stability and similarly reduced systemic toxicity (12). This allows for reduction of side effects at a given dose, but also potentially for larger doses of chemotherapy to be given. Liposomes were prepared as described $(40,52)$ and are summarized in the Supplemental Methods.

Tumor generation. Female FVB/n mice (6 to 10 weeks old, 15-25 g, Charles River) (Supplemental Table 1) were transplanted with fresh donor biopsy pieces of NDL syngeneic mammary carcinoma $\left(\mathrm{ca} .1 \mathrm{~mm}^{3}\right)$; standard techniques were used, and NDL mammary carcinomas were derived as previously described (53). Approximately 3 weeks later, mice were divided into treatment groups using a tumor size-stratified random sample, with tumor size ranging from 4 to $6 \mathrm{~mm}$ in the longest dimension as measured by ultrasound (Sequoia, Siemens Preclinical Solutions).

The NDL cell line was developed within the Borowsky laboratory (37). $4 \mathrm{~T} 1$ cells were purchased from ATCC, with additional details of tumor generation described in Supplemental Methods.

MRgFUS and CET1wMRI protocols. MRgFUS was performed with a Bruker BioSpec 7T small animal system (Bruker Biospin), MRcompatible 16-element annular transducer (Imasonic SAS), $3 \mathrm{MHz}$ central frequency, $300 \mathrm{kHz}$ bandwidth, $120 \mathrm{~W}$ peak acoustical power (where 5 and 10 acoustic Watts were used in this study), $85^{\circ}$ aperture, $48 \mathrm{~mm}$ diameter, $35 \mathrm{~mm}$ radius of curvature, $0.5 \mathrm{~mm} \times 0.5 \mathrm{~mm} \times 1.5$ $\mathrm{mm}$ full width half max focus size, and MR-compatible transducer positioning system (Image Guided Therapy). Without loss of generality, a $3 \mathrm{MHz}$ center frequency was selected to provide a narrow focus and a short axial focal length in order to compare drug delivery augmentation for single-point, grid, and scanned protocols in a mouse model. Temperature was monitored by proton resonance frequency shift with Thermoguide software (Image Guided Therapy), with $\alpha=$ $-0.0101 \mathrm{ppm} /{ }^{\circ} \mathrm{C}, \mathrm{TE} / \mathrm{TR}=4.5 / 21 \mathrm{~ms}$, calibrated as described (54). Acoustic pressure was calibrated directly with a fiber optic hydrophone (HFO690, Onda Corp.) in a degassed water bath in free-field conditions. Temperature was validated with a Luxtron STB fiber optic probe (Lumasense Technologies). Simulation of heating used a finite-difference model based on Pennes' bioheat transfer equation described in Supplemental Methods. Scan parameters of TE $/ \mathrm{TR}=$ $12.5 / 750 \mathrm{~ms}, \mathrm{FOV}=3.2 \mathrm{~cm} \times 3.2 \mathrm{~cm}, \mathrm{MTX}=256 \times 256, \mathrm{ST} / \mathrm{SI}=1 / 1$ $\mathrm{mm}$, and 9 slices were used for T1-weighted imaging. Prior to MRgFUS, mice were given $0.05-0.1 \mathrm{mg} / \mathrm{kg}$ buprenorphine s.c. and 0.05 $\mathrm{mmol} / \mathrm{kg}$ of gadoteridol (Bracco Imaging) via i.p. injection. We compared i.p. and i.v. administration as well as injection before and after ablation with no injection or no ultrasound as control protocols. No significant differences in distribution were observed. As i.p. injection allows for sustained tumor contrast enhancement during the ablation procedure, we used i.p. injection for CET1wMRI immediately following the ablation procedure, then used i.v. injection for imaging at later time points.

Mice were first imaged with T1wMRI for tumor localization. Tumors were either ablated at a single point in the center of the tumor as identified by T1wMRI, in a grid pattern covering the entire tumor volume with points separated by $1.5 \mathrm{~mm}$, or in a circular pattern with a diameter of $2 \mathrm{~mm}$ and scan speed of 1 revolution per second. The grid pattern protocol included a minimum of 30 seconds of cooling time between ultrasound exposures, with a separate acquisition of thermometry for each exposure. As a typical tumor received 15 points of thermal ablation, the time required for the circular protocol was 15 -fold less than the grid protocol (1 minute vs. 15 minutes), excluding the time required for animal handling and MR imaging. Each application was designed to induce temperatures greater than $65^{\circ} \mathrm{C}$ and a CEM43 of more than 5,000. For the single-spot and grid protocols, we initially compared a 7-second continuous wave (CW) application of 10 acoustic watts (7.6 MPa PNP) with a 20-second CW application of 5 acoustic watts (3.1 MPa PNP) with no significant difference observed in temperature or accumulation; therefore, data from these settings are combined. We chose a 60-second CW application of 5 acoustic watts (3.1 MPa PNP) for the circular protocol.

For hyperthermia used with TSL, the system and protocol previously described (12) were applied. One tumor per animal was insonified for 5 minutes at $42^{\circ} \mathrm{C}$ prior to administration of drug; the tumor insonation was continued for an additional 20 minutes at $42^{\circ} \mathrm{C}$ after injection. The ultrasound pulses consisted of 100-cycle bursts at 1.5 $\mathrm{MHz}$ center frequency and $1.1 \mathrm{MPa} \mathrm{PNP}$, with a variable pulse-repetition frequency ranging from $100 \mathrm{~Hz}$ up to $5 \mathrm{kHz}(12)$.

PET-CT imaging, $\gamma$ count, histology, and autoradiography protocols. Standard methods were used as previously described $(52,55)$. Briefly, all PET imaging was performed with 2 mice side by side, using a microPET Focus 120 scanner (Siemens Preclinical Solutions) and Siemens Inveon CT scanner. Mice were imaged for 0.5 hours at each time point $(3,20$, and 48 hours) following injection. PET images were reconstructed with maximum a posteriori algorithm, and tumors were manually segmented with PET contrast normalized to 0 and 25 \%ID/cc by a single observer with Image (http://imagej.nih.gov/ij/), ASIPro, and Inveon Research Workplace software (Siemens Preclinical Solutions). Activity measured within 3D volumes was time decay corrected and expressed as \%ID/cc. Due to the limitations of the PET isotopes here, the $\mathrm{AUC}_{48}$ was calculated using a trapezoidal approximation over the 48 hours of data collected, which underestimates the full AUC, as the clearance of the radiotracer is less than $10 \%$ of the peak at 48 hours in clinically relevant grid and circle protocols. 
Following imaging, mice were euthanized by cervical dislocation; organs of interest were harvested and weighed, and radioactivity was measured by Wizard 1470 Automatic Gamma Counter (PerkinElmer). Tumors were either fixed with $20 \%$ neutral buffered formalin, embedded in paraffin, and stained with H\&E or frozen in OCT compound (Tissue-Tek) with dry ice and isopropanol and sectioned; serial sections were either stained with H\&E or used to develop a storage phosphor screen overnight, which was then read with a Storm 860 phosphor imaging system (GE Healthcare).

Biodistribution of liposomes and gadoteridol. To evaluate liposome biodistribution, NDL tumor-bearing FVB/n mice were treated once with MRgFUS ablation (single point, circle, grid, or control no ultrasound), injected with ${ }^{64} \mathrm{Cu}-\mathrm{LCL}$ (i.v., $1 \mathrm{mg}$ lipid/mouse), imaged with PET-CT and CET1wMRI at intervals up to 48 hours, then sacrificed for $\gamma$ count, histology, and autoradiography (Figure 1A). A second cohort was treated once with ablation (circle or control no ultrasound) and injected with gadoteridol (i.v. or i.p. and before or after ablation) and imaged with CET1wMRI at intervals up to 48 hours. A third cohort was treated once with MRgFUS ablation (single point, circle, grid, or control no ultrasound) and then injected with A555-LCL (i.v., $1 \mathrm{mg}$ lipid/mouse). These mice were then sacrificed at 24 hours, and tumors were harvested for optical imaging and viability studies. A fourth cohort of mice was treated with weekly ablation (single point or control no ultrasound) and biweekly Dox-LCL (i.v., $6 \mathrm{mg}$ / $\mathrm{kg}$ ) for 2 weeks, then treated with ablation (or control no ultrasound) and injected with ${ }^{64} \mathrm{Cu}$-LCL. These mice were then imaged with PET-CT at intervals up to 48 hours and sacrificed for necropsy and biodistribution. Additional details are provided in the Supplemental Methods.

Survival studies. To evaluate survival following MRgFUS ablation and Dox-LCL chemotherapy, 6 groups of NDL tumor-bearing mice were treated weekly with MRgFUS single-point ablation $(n=8)$, circle protocol $(n=4)$, single-point ablation plus Dox-LCL $(n=4)$, circle ablation plus Dox-LCL $(n=4)$, control (no ultrasound and no drug) $(n=8)$, or Dox-LCL only $(n=4)$. Mice were injected biweekly with Dox-LCL (or control saline) for a period of 4 weeks ( 4 ablations and 8 injections total). Dox-LCL (i.v., 6 $\mathrm{mg} / \mathrm{kg}$ ) was injected in the tail vein immediately after ablation.

To evaluate survival following MRgFUS ablation and Dox-TSL chemotherapy, 4 groups of NDL tumor-bearing mice were treated weekly with MRgFUS ablation (single-point ablation plus Dox-TSL $(n=4)$, circle ablation plus Dox-TSL $(n=4)$, circle ablation preceded by hyperthermia and Dox-TSL $(n=4)$, no treatment (no ultrasound and no drug) $(n=4)$, or Dox-TSL only $(n=4)$ for a period of 4 weeks. DoxTSL (i.v., $6 \mathrm{mg} / \mathrm{kg}$ ) was injected in the tail vein immediately prior to ablation in order to release the drug during ablation. In the Dox-TSL plus hyperthermia group, hyperthermia was established for 5 minutes, Dox-TSL was injected, hyperthermia was continued for 20 minutes, and ablation was performed immediately after hyperthermia.

In each case, mice were sized with ultrasound biweekly and sacrificed when tumors reached $2 \mathrm{~cm}$ in the longest dimension.

Statistics. Results are presented as mean \pm SEM. One-way ANOVA (Microsoft Excel 2010 with Daniel's XL toolbox version 6.53) was used to compare $\mathrm{AUC}_{48}$ among contralateral tumors and untreated tumors, and then 1-way ANOVA followed by Tukey's test was used to compare $\mathrm{AUC}_{48}$ among MRgFUS-treated tumors and untreated tumors. $P$ values of less than 0.05 were considered significant.

Study approval. All animal studies were approved by the UCD Institutional Animal Care and Use Committee.

\section{Author contributions}

AWW, KDW, LMM, and KWF designed research studies. AWW, BZF, YL, AK, JWS, KDW, LMM, ST, HZ, and JF performed research. AK, JWS, JF, and $\mathrm{ADB}$ contributed reagents and analytic tools. AWW, ADB, and KWF analyzed data. AWW and KWF wrote the paper.

\section{Acknowledgments}

We gratefully acknowledge the support of NIHR01CA134659, NIHR01CA199658, and NIHR01CA103828. We also thank Sandra Taylor and Susan Stewart of the Center for Translational Medicine at UCD for expert advice in statistical analysis.

Address correspondence to: Katherine W. Ferrara, Department of Biomedical Engineering, University of California, Davis, 451 Health Sciences Drive, Davis, California 95616, USA. Phone: 530.754.9436; E-mail: kwferrara@ucdavis.edu.
1. Alm El-Din MA, Taghian AG. Breast conservation therapy for patients with locally advanced breast cancer. Semin Radiat Oncol. 2009;19(4):229-235

2. Gralow JR, et al. Preoperative therapy in invasive breast cancer: pathologic assessment and systemic therapy issues in operable disease. J Clin Oncol.2008;26(5):814-819.

3. Weinberg BD, Krupka TM, Haaga JR, Exner AA. Combination of sensitizing pretreatment and radiofrequency tumor ablation: evaluation in rat model. Radiology. 2008;246(3):796-803.

4. Ahmed M, et al. Combination radiofrequency ablation with intratumoral liposomal doxorubicin: effect on drug accumulation and coagulation in multiple tissues and tumor types in animals. Radiology. 2005;235(2):469-477.

5. Ahmed M, et al. Radiofrequency thermal ablation sharply increases intratumoral liposomal doxorubicin accumulation and tumor coagulation. Cancer Res. 2003;63(19):6327-6333.

6. Ahmed M, Goldberg SN. Combination radiofrequency thermal ablation and adjuvant IV liposomal doxorubicin increases tissue coagulation and intratumoural drug accumulation. Int J Hyperthermia. 2004;20(7):781-802.

7. Ikink ME, et al. Volumetric MR-guided highintensity focused ultrasound versus uterine artery embolisation for treatment of symptomatic uterine fibroids: comparison of symptom improvement and reintervention rates. Eur Radiol. 2014;24(10):2649-2657.

8. Tempany CM, Stewart EA, McDannold N, Quade BJ, Jolesz FA, Hynynen K. MR imaging-guided focused ultrasound surgery of uterine leiomyomas: a feasibility study. Radiology. 2003;226(3):897-905

9. Galiana G, Branca RT, Jenista ER, Warren WS. Accurate temperature imaging based on intermolecular coherences in magnetic resonance. Science. 2008;322(5900):421-424.

10. Quesson B, de Zwart JA, Moonen CTW. Magnetic resonance temperature imaging for guidance of thermotherapy. J Magn Reson Imaging. 2000;12(4):525-533.

11. Dewhirst MW, Vujaskovic Z, Jones E, Thrall D.
Re-setting the biologic rationale for thermal therapy. Int J Hyperthermia. 2005;21(8):779-790.

12. Kheirolomoom A, et al. Complete regression of local cancer using temperature-sensitive liposomes combined with ultrasound-mediated hyperthermia. JControl Release. 2013;172(1):266-273.

13. Chida S, Okada K, Suzuki N, Komori C, Shimada Y. Infiltration by macrophages and lymphocytes in transplantable mouse sarcoma after irradiation with high-intensity focused ultrasound. Anticancer Res. 2009;29(10):3877-3882.

14. Liu F, et al. Boosting high-intensity focused ultrasound-induced anti-tumor immunity using a sparsescan strategy that can more effectively promote dendritic cell maturation. J Transl Med.2010;8:7.

15. Seo JW, Qin S, Mahakian LM, Watson KD, Kheirolomoom A, Ferrara KW. Positron emission tomography imaging of the stability of $\mathrm{Cu}-64$ labeled dipalmitoyl and distearoyl lipids in liposomes. J Control Release. 2011;151(1):28-34.

16. Kong G, Braun RD, Dewhirst MW. Characterization of the effect of hyperthermia on nanoparti- 
cle extravasation from tumor vasculature. Cancer Res. 2001;61(7):3027-3032.

17. Yuan F, Chen Y, Dellian M, Safabakhsh N, Ferrara N, Jain RK. Time-dependent vascular regression and permeability changes in established human tumor xenografts induced by an anti-vascular endothelial growth factor/vascular permeability factor antibody. Proc Natl Acad Sci US A. 1996;93(25):14765-14770.

18. Yuan F, Leunig M, Huang SK, Berk DA, Papahadjopoulos D, Jain RK. Microvascular permeability and interstitial penetration of sterically stabilized (stealth) liposomes in a human tumor xenograft. Cancer Res. 1994;54(13):3352-3356.

19. Monsky WL, et al. Augmentation of transvascular transport of macromolecules and nanoparticles in tumors using vascular endothelial growth factor. Cancer Res. 1999;59(16):4129-4135.

20. Infanger M, Schmidt O, Kossmehl P, Grad S, Ertel W, Grimm D. Vascular endothelial growth factor serum level is strongly enhanced after burn injury and correlated with local and general tissue edema. Burns. 2004;30(4):305-311.

21. Watson KD, et al. Ultrasound increases nanoparticle delivery by reducing intratumoral pressure and increasing transport in epithelial and epithelial-mesenchymal transition tumors. Cancer Res. 2012;72(6):1485-1493.

22. Dekanski J. The effect of cutaneous burns on histamine in mice. J Physiol. 1945;104(2):151-160.

23. Lund T, Onarheim H, Reed RK. Pathogenesis of edema formation in burn injuries. World J Surg. 1992;16(1):2-9.

24. Karino T, Koga S, Maeta M. Experimental studies of the effects of local hyperthermia on blood flow, oxygen pressure and $\mathrm{pH}$ in tumors. Jpn J Surg. 1988;18(3):276-283.

25. Urano M, Kuroda M, Nishimura Y. For the clinical application of thermochemotherapy given at mild temperatures. Int J Hyperthermia. 1999;15(2):79-107.

26. Issels RD. Hyperthermia adds to chemotherapy. Eur J Cancer. 2008;44(17):2546-2554.

27. Fry WJ, Wulff VJ, Tucker D, Fry FJ. Physical factors involved in ultrasonically induced changes in living systems. 1. Identification of non-temperature effects. JAcoust Soc Am. 1950;22(6):867-876.

28. Wang S, et al. Pulsed high intensity focused ultrasound increases penetration and therapeutic efficacy of monoclonal antibodies in murine xenograft tumors. JControl Release. 2012;162(1):218-224.

29. Yuh EL, et al. Delivery of systemic chemotherapeutic agent to tumors by using focused ultrasound: study in a murine model. Radiology. 2005;234(2):431-437.

30. Mu ZM, Ma CM, Chen XM, Cvetkovic D, Pollack A, Chen LL. MR-guided pulsed high intensity focused ultrasound enhancement of docetaxel combined with radiotherapy for prostate cancer treatment. Phys Med Biol. 2012;57(2):535-545.

31. Caskey CF, Qin SP, Dayton PA, Ferrara KW. Microbubble tunneling in gel phantoms. J Acoust Soc Am. 2009;125(5):El183-ElEl9.

32. Immordino ML, Dosio F, Cattel L. Stealth liposomes: review of the basic science, rationale, and clinical applications, existing and potential. Int $J$ Nanomedicine. 2006;1(3):297-315.

33. Maeda H. The enhanced permeability and retention (EPR) effect in tumor vasculature: the key role of tumor-selective macromolecular drug targeting. Adv Enzyme Regul. 2001;41:189-207.

34. Dromi S, et al. Pulsed-high intensity focused ultrasound and low temperature-sensitive liposomes for enhanced targeted drug delivery and antitumor effect. Clin Cancer Res. 2007;13(9):2722-2727.

35. Staruch RM, Ganguly M, Tannock IF, Hynynen K, Chopra R. Enhanced drug delivery in rabbit VX2 tumours using thermosensitive liposomes and MRI-controlled focused ultrasound hyperthermia. Int J Hyperthermia. 2012;28(8):776-787.

36. Andriyanov AV, Koren E, Barenholz Y, Goldberg SN. Therapeutic efficacy of combining pegylated liposomal doxorubicin and radiofrequency $(\mathrm{RF})$ ablation: comparison between slow-drug-releasing, non-thermosensitive and fast-drug-releasing, thermosensitive nano-liposomes. PLoS One. 2014;9(5):e92555.

37. Cardiff RD, et al. Quantitation of fixativeinduced morphologic and antigenic variation in mouse and human breast cancers. Lab Invest. 2013;93(4):480-497.

38. Miller JK, et al. Suppression of the negative regulator LRIG1 contributes to ErbB2 overexpression in breast cancer. Cancer Res. 2008;68(20):8286-8294.

39. May JP, Li SD. Hyperthermia-induced drug targeting. Expert Opin Drug Deliv. 2013;10(4):511-527.

40. Kheirolomoom A, et al. Copper-doxorubicin as a nanoparticle cargo retains efficacy with minimal toxicity. Mol Pharm. 2010;7(6):1948-1958.

41. Laginha KM, Verwoert S, Charrois GJ, Allen TM. Determination of doxorubicin levels in whole tumor and tumor nuclei in murine breast cancer tumors. Clin Cancer Res. 2005;11(19 pt 1):6944-6949.

42. Torigian DA, et al. PET/MR imaging: technical aspects and potential clinical applications. Radi- ology. 2013;267(1):26-44.

43. Kohler MO, et al. Volumetric HIFU ablation under 3D guidance of rapid MRI thermometry. Med Phys. 2009;36(8):3521-3535.

44. Gianfelice D, Khiat A, Amara M, Belblidia A, Boulanger Y. MR imaging-guided focused US ablation of breast cancer: histopathologic assessment of effectiveness - initial experience. Radiology. 2003;227(3):849-855.

45. McDannold, Hynynen K, Wolf D, Wolf G, Jolesz F. MRI evaluation of thermal ablation of tumors with focused ultrasound. J Magn Reson Imaging. 1998;8(1):91-100.

46. Ho CS, Ju KC, Cheng TY, Chen YY, Lin WL. Thermal therapy for breast tumors by using a cylindrical ultrasound phased array with multifocus pattern scanning: a preliminary numerical study. Phys Med Biol. 2007;52(15):4585-4599.

47. Haar GT, Coussios C. High intensity focused ultrasound: physical principles and devices. Int $J$ Hyperthermia. 2007;23(2):89-104.

48. Lu J, Hu W, Wang W. Sonablate-500 transrectal high-intensity focused ultrasound (HIFU) for benign prostatic hyperplasia patients. J Huazhong Univ Sci Technolog Med Sci. 2007;27(6):671-674.

49. Li T, et al. Pulsed high-intensity focused ultrasound enhances delivery of doxorubicin in a preclinical model of pancreatic cancer. Cancer Res. 2015;75(18):3738-3746.

50. Staruch R, Chopra R, Hynynen K. Localised drug release using MRI-controlled focused ultrasound hyperthermia. Int J Hyperthermia. 2011;27(2):156-171.

51. Feng M, Yang YL, He PG, Fang YZ. Spectroscopic studies of copper(II) and iron(II) complexes of adriamycin. Spectrochim Acta A Mol Biomol Spectrosc. 2000;56(3):581-587.

52. Seo JW, Zhang H, Kukis DL, Meares CF, Ferrara KW. A novel method to label preformed liposomes with 64Cu for positron emission tomography (PET) imaging. Bioconjug Chem. 2008;19(12):2577-2584.

53. Damonte P, Hodgson JG, Chen JQ, Young LJ, Cardiff RD, Borowsky AD. Mammary carcinoma behavior is programmed in the precancer stem cell. Breast Cancer Res. 2008;10(3):R50.

54. Fite BZ, et al. Magnetic resonance thermometry at $7 \mathrm{~T}$ for real-time monitoring and correction of ultrasound induced mild hyperthermia. PLoS One. 2012;7(4):e35509.

55. Son DJ, et al. The atypical mechanosensitive microRNA-712 derived from pre-ribosomal RNA induces endothelial inflammation and atherosclerosis. Nat Commun. 2013;4:3000. 